\title{
Signal To Noise Ratio Mapping For Cryo-Electron Tomography
}

\author{
C. Renken
}

Resource for the Visualization of Biological Complexity, Wadsworth Center, Empire State Plaza, PO. Box 509 Albany, NY 12201-0509

Recently during experiments involving template matching of Ryanodine receptors within cryoelectron tomograms [1], we noticed that the quality of identified Ryanodine receptors varied randomly within the tomogram. While it was difficult to directly attribute this to features within the tomogram, Ryanodine receptors near added colloidal gold particles generally were the worst in terms of reconstruction quality. Obviously, for an equivalent ice thickness an isolated particle will reconstruct with greater fidelity than a particle within a crowded environment. For an isolated

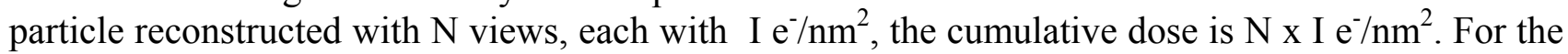
particle within the crowd, a significant number of views contains $k_{i}$ added particles. Assuming the weak phase approximation these $k_{i}$ added particles induce an added phase shift beyond that of an isolated particle. During back-projection the signal of this projection for the primary particle is now $1 /\left(k_{i}+1\right)$. The noise due to the obstructing particles is then $k_{i} /\left(k_{i}+1\right)$. Thus while the cumulative dose used to reconstruct a single particle in a crowded environment is the same only a fraction of the total dose

$$
\sum_{i=1}^{\mathrm{N}} \frac{\mathrm{I}}{k_{i}+1} \leq \mathrm{Nx} \mathrm{I}
$$

is signal and the signal to noise ratio (SNR) at the particle is

$$
\sum_{i=1}^{\mathrm{N}}\left(\frac{1}{k_{i}}\right)
$$

Since each particle within a tomogram will have its own set of $k_{i}$ the SNR for each particle will obviously differ.

We therefore propose to calculate the SNR at each voxel within the tomogram. based upon the variance between the contributing pixels in the projections. For each voxel within the tomogram we define the SNR as the average projected intensity divide by the standard deviation in projected intensities for that voxel or

$$
\mathrm{SNR}_{\mathrm{ijk}}=\frac{\sum_{\mathrm{n}=1}^{\mathrm{N}}\left(\mathrm{P}_{\mathrm{i}^{\prime} j^{\prime} \mathrm{n}}\right)}{\sqrt{N \sum_{\mathrm{n}=1}^{\mathrm{N}}\left(\mathrm{P}_{\mathrm{i}^{\prime} j^{\prime} \mathrm{n}}-\overline{\mathrm{P}}_{\mathrm{i} \mathrm{j}^{\prime} \mathrm{j}^{\prime}}\right)^{2}}}
$$

where $P$ is the intensity at pixel(i',j') of projection $n$ that contributes to voxel $(\mathrm{i}, \mathrm{j}, \mathrm{k})$ of the volume. Figure 1 shows the point spread function (PSF) of a $\pm 60^{\circ}$ tomogram (a,b,c,d,e) and the SNR map (f,g,h,j,k) of the corresponding PSF. The PSF of tomograms are characterized by streaking along the 
sampled directions. This artifact is due to the additive nature of the reconstruction algorithms. Each projection projects a finite density completely through the reconstruction into voxels that should contain zero density. Fortunately these voxels are sampled by other projections which contribute zero density. Thus the variance between contributing projections becomes large. This results in low SNR values in the regions prone to artifacts. Direct weighting of the voxels of the PSF tomogram with their SNR value suppresses these artifacts (figure $1 \mathrm{k}, 1, \mathrm{~m}, \mathrm{n}, \mathrm{o}$ ).

We propose the SNR map as a boot strap method to estimate the local uncertainty within tomograms. Areas containing high SNR values are likely free of artifacts and may be interpreted with finer resolution. Areas of low SNR values are prone to artifacts and high resolution features should be suspect. By using the SNR map of the tomogram to get a local measure of resolution, rather than an absolute global measure of resolution, the researcher is free to apply a priori knowledge of the sample when interpreting features of interest. Thus the resolution question becomes a local probability of discerning features given the quality of the data and any prior knowledge of the researcher.

\section{References}

[1] C. Renken et al. J. Struct. Biol. 165 (2009) 53.

[2] This work was supported by NIH NCRR Grant P41 RR01219 (P.I.: C. Mannella)
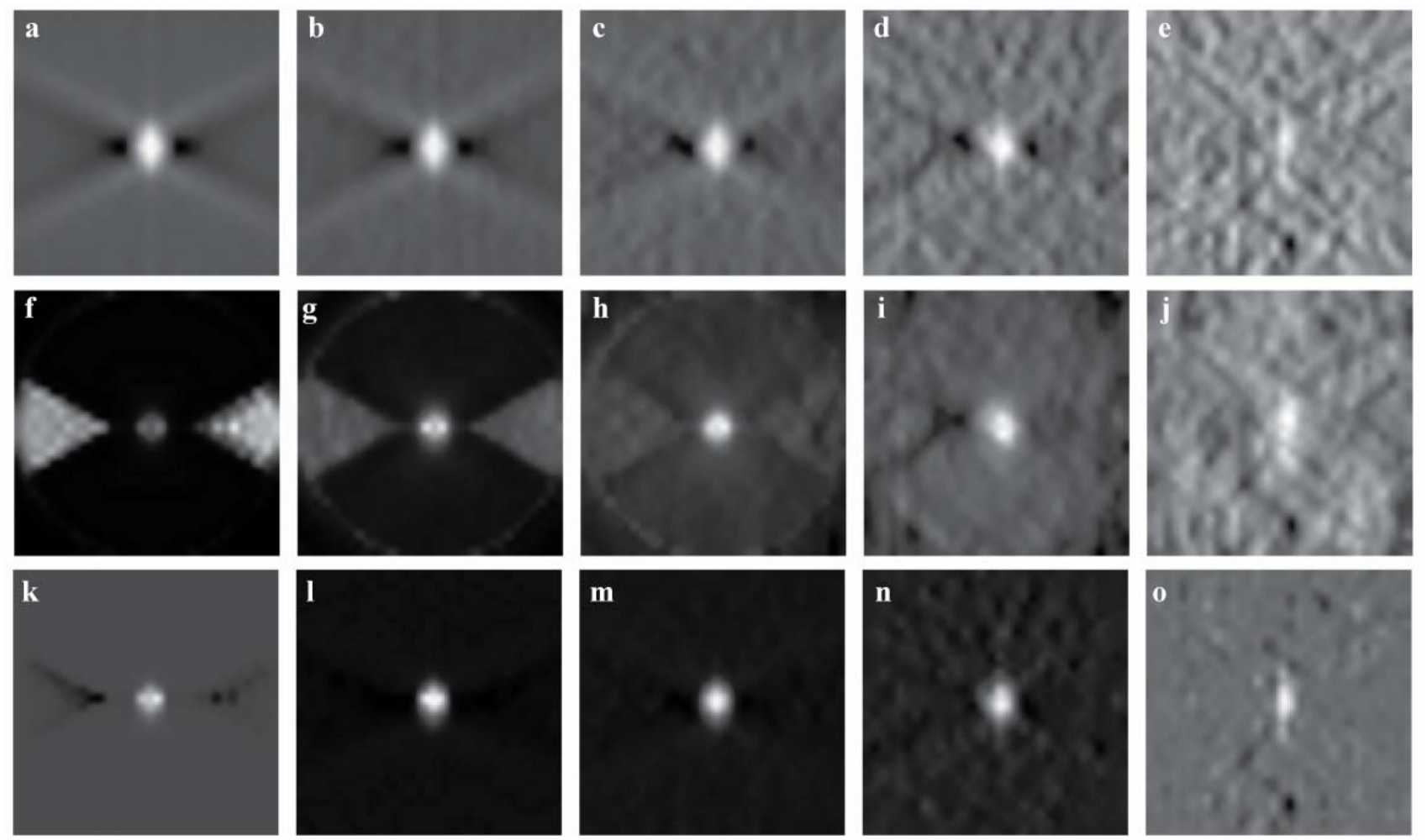

Figure. Point spread function of a tomogram sampled form $+60^{\circ}$ to $-60^{\circ}$ at $5^{\circ}$ increments (a-e), the corresponding SNR map ( $\mathrm{f}-\mathrm{j})$ and the weighted PSF (k-o). Guassian noise is added to the projections at a SNR of $10^{6},(\mathrm{a}, \mathrm{f}, \mathrm{k}), 10^{2}(\mathrm{~b}, \mathrm{~g}, \mathrm{l}), 10^{1}(\mathrm{c}, \mathrm{h}, \mathrm{m}), 10^{0}(\mathrm{~d}, \mathrm{i}, \mathrm{n})$, and $10^{-1}(\mathrm{e}, \mathrm{j}, \mathrm{o})$. Individual boxes are $32 \mathrm{x}$ 32 pixels. 